\title{
Diversidade Inventiva e Hierarquia Urbana no Brasil
}

\author{
Suelen Sales $^{1}$ (D) | Eduardo Gonçalves ${ }^{2}$ (D) | Rodrigo Rodriguez ${ }^{2}$ (iD \\ ${ }^{1}$ Universidade Federal de Juiz de Fora. E-mail: sususales13@hotmail.com \\ ${ }^{2}$ Universidade Federal de Juiz de Fora. E-mail: eduardo.goncalves@ufjf.edu.br \\ ${ }^{3}$ Universidade Federal Fluminense. E-mail: rrodriguez431@gmail.com
}

\begin{abstract}
RESUMO
A Teoria dos Lugares Centrais de Christaller concebe sistemas de cidades em que a quantidade de funções aumenta à medida que se expande o tamanho desses centros urbanos. Este artigo explora a diversidade inventiva no Brasil por meio das classes tecnológicas dos dados de patentes do Instituto Nacional da Propriedade Industrial (INPI) e sua relação com a hierarquia urbana, tendo como base o conceito de Regiões de Influência das Cidades (REGIC), no período entre 2000 e 2011. Além da maior quantidade de depósitos de patentes, as regiões de maior porte realizam não apenas as funções características de áreas menores, mas também as invenções menos comuns. Os resultados são consistentes com a organização hierárquica do sistema de cidades, sinalizando que a posição na hierarquia urbana pode ser tão relevante na determinação das categorias de invenção quanto os aspectos cognitivos, institucionais e espaciais.
\end{abstract}

\section{PALAVRAS-CHAVE}

Invenção, Classes de patentes, Diversidade técnica, Hierarquia urbana, REGIC

\section{Inventive Diversity and Urban Hierarchy in Brazil}

\begin{abstract}
Christaller's Theory of Central Places conceives systems of cities in which functions increase as the size of these urban centers expands. This article explores the inventive diversity in Brazil through the patent technological codes from the National Institute of Industrial Property (INPI) and its relationship with the urban hierarchy, based on the concept of Regions of Influence of Cities (REGIC), for the period between 2000 and 2011. In addition to the greater number of patent applications, the larger regions perform not only the functions that are characteristic of smaller areas, but also the less common inventions, with a positive relationship between creative diversity and urban size. This indicates that the hierarchical organization of the city system can be as relevant in determining the categories of invention as the cognitive, institutional, and spatial aspects.
\end{abstract}

\section{KEYWORDS}

Invention, Patent classes, Technical diversity, Urban hierarchy, REGIC

\section{CLASSIFICAÇÃO JEL}

O30, R10, D39

\section{Introdução}

O processo inventivo depende do conhecimento tecnológico capaz de ser absorvido e processado pelas pessoas, tendo o aprendizado tecnológico um caráter cumulativo (Berger e Luckmann, 1966). Ao nível regional, a capacidade inventiva pode ser avaliada por meio de suas características locais, a partir de medidas como a densidade e a 
escala urbana (Christaller, 1966). Além disso, outros elementos como a proximidade entre os agentes, sua localização, a infraestrutura urbana e de pesquisa científica e tecnológica são fatores destacados pela literatura, além de serem mais ofertados nos grandes centros urbanos (Howells, 2002; Feldman e Florida, 1994). Dessa forma, a localização geográfica e a maior interação entre os agentes constituem-se mecanismos de transferência importantes que podem originar novos usos, novas soluções e aperfeiçoamento de técnicas já existentes, favorecendo o processo inventivo Scott (2009). Regiões mais bem posicionadas na hierarquia urbana podem assumir a dianteira no processo de crescimento regional, criando processos desiguais no espaço.

A partir do conceito de hierarquia urbana de Christaller (1966) e seu debate reconduzido por Krugman (1996), é possivel discutir se há uma relação entre a distribuição regional da atividade inventiva e a escala urbana dessas regiões, a partir da avaliação dos efeitos da estrutura de hierarquia urbana. Uma das hipóteses avaliadas a partir dessa teoria é a de que centros urbanos de maior porte são localidades com maior número de invenções (i.e., quantitativo) e invenções mais sofisticadas (i.e., qualitativo), caracterizando áreas de maior diversidade e potencialidade criativa tanto em termos absolutos como em termos relativos. Nesse sentido, os centros urbanos podem ser compreendidos como localidades dotadas de variedade inventiva e escalar diferenciadas, sendo entendidas como forças "centrípetas" atrativas à alocação dos fatores inventivos nessas regiões (McCann, 2014). O estudo da concentração regional das invenções e de sua respectiva natureza hierárquica dota os formuladores de política econômica das ferramentas necessárias para o planejamento do crescimento regional.

Para o caso do Brasil, há estudos correlacionando a hierarquia urbana e a atividade inventiva, por exemplo, nas estimações a partir de análises empíricas apresentadas por Ruiz (2005); Simões et al. (2010). Em geral, os estudos para o Brasil convergem sobre a predominância das regiões Sul-Sudeste nas invenções brasileiras, nas quais se concentra a maior parte da atividade inventiva do Brasil. Montenegro et al. (2011) ressaltam que há fatores condicionantes para a perpetuação dessas regiões mais inovadoras frente àquelas regiões de menor atividade tecnológica, designados como efeitos inerciais temporais e espaciais sobre a inovação, ou seja, há uma defasagem decorrente da própria capacidade tecnológica regional que propicia a manutenção das desproporções entre as regiões no que se refere à inovação.

Araujo et al. (2016), também sobre o caso brasileiro, confirmam hipóteses sobre a existência de transbordamentos e externalidades advindas da diversificação da atividade econômica. Nesse estudo, os autores reconhecem o papel da escala urbana como um fator condicionante expressivo para a inovação regional. Nesse sentido, a escala urbana está associada à constituição de redes sociais de desenvolvimento tecnológico nos maiores centros urbanos, induzindo mais profissionais a se envolverem em atividades inventivas e atraindo profissionais qualificados para essas regiões (Bettencourt, 2013).

Para além da concentração espacial da atividade inventiva brasileira, Rodriguez e 
Gonçalves (2017) argumentam por meio de modelos empíricos com base em dados de patentes que há uma forte associação entre a escala urbana e o grau de sofisticação tecnológica das atividades inventivas. Essas tecnologias sofisticadas, como as tecnologias de engenharia elétrica, telecomunicações e nanotecnologia, concentram-se em São Paulo, Campinas e Rio de Janeiro e são potencializadas pela capacidade dos grandes centros urbanos em centralizar esses tipos de atividades.

O presente artigo pretende contribuir para essa discussão ao analisar a distribuição da atividade inventiva no Brasil, sua diversidade tecnológica e como as invenções se relacionam com o conceito de hierarquia urbana, seguindo a metodologia empregada por Ó hUallacháin et al. (2015) para a economia norte americana, aplicando-a para uma economia distante da fronteira tecnológica. Para isso, foram utilizados dados de patentes do Instituto Nacional da Propriedade Industrial (INPI) como proxy para a atividade inventiva, agregados para as Regiões de Influência das Cidades (REGIC), que identificam as metrópoles e capitais regionais brasileiras e seu alcance espacial de influência, para o período entre 2000 e 2011.

Pretende-se, assim, analisar empiricamente o papel desempenhado pelo espaço urbano para a capacidade inventiva de uma região. O artigo tem como objetivo explorar a diversidade da especialização tecnológica das regiões brasileiras, a partir da relação entre o nível de centralidade de determinada região, mensurada pelo seu porte, e sua função dentro da hierarquia urbana de inovação. Diante dessas análises, compararse-ão as conclusões entre os resultados obtidos para os casos dos Estados Unidos e do Brasil, ponderando se há os mesmos efeitos de escala urbana em um país desenvolvido e em um subdesenvolvido no tocante à distribuição espacial das invenções.

Este artigo está subdividido em seis seções, além desta introdução. Na segunda seção, aborda-se a relação entre inovação e centralidade urbana. Na terceira e quarta seção, são descritas a base de dados e a metodologia utilizadas no trabalho, respectivamente. Na quinta seção, são apresentados os resultados da pesquisa. Por fim, na sexta seção, são apresentadas as conclusões.

\section{Revisão de literatura e hipóteses}

O caráter regional da invenção, isto é, a maneira como a atividade inventiva se concentra em determinadas regiões, é um debate pertinente para a teoria econômica cujo principal objetivo é explicar as razões pelas quais a produção de tecnologia e as atividades produtivas se concentram em localidades específicas. Segundo Simmie (2001), o processo de inovação se constitui em um conjunto de processos coletivos e dinâmicos que incorporam diversos fatores dentro de uma determinada região, as quais produzem redes de inter-relações sinérgicas. As oportunidades para o desenvolvimento de tecnologias, mesmo que heterogêneas, existem para todas as regiões, porém as evidências advindas das economias urbanas concentradas em regiões específicas sugerem que há uma diferença com que cada região é capaz de mobilizar seus 
recursos a fim de extrair plenamente seu potencial de crescimento (Simmie, 2001).

A concentração da atividade inventiva ocorre pelas variadas potencialidades que uma localidade é capaz de oferecer aos agentes. As invenções são propiciadas, por exemplo, pela existência de um mercado de trabalho especializado, por inter-relações entre fornecedores e compradores regionais e por contatos face a face que promovem os spillovers de conhecimento, sendo todos facilitados pela aproximação espacial, conferindo características distintas a cada região (Marshall, 1985; Audretsch e Feldman, 1996). As regiões que comportam maior diversidade em sua estrutura industrial tendem a ter maiores niveis de inovação, devido à presença de diferentes indústrias que permitem a reconfiguração do conhecimento adquirido, aumentando a propensão dos indivíduos em desenvolver produtos novos e diferenciados (Jacobs, 1969).

Com a literatura da Nova Geografia Econômica (NGE) e sua ênfase na modelagem dos processos de aglomeração das atividades econômicas para as mais diversas escalas regionais, uma das principais sínteses por Fujita e Mori (2005) é a existência de uma hierarquia urbana estável na maior parte dos países do seguinte tipo: cidades de médio porte se especializando em um número limitado de indústrias e grandes metrópoles com indústrias altamente diversificadas que não são diretamente relacionadas. Com a preocupação direcionada para as atividades inventivas, a geografia da atividade inventiva é concentrada e localizada nos centros urbanos, beneficiada pelos burburinhos locais (local buzz) e pelas redes globais (global pipelines) (Feldman e Kogler, 2010).

As hierarquias urbanas, em particular aquelas especialmente inovadoras, têm sido extremamente persistentes ao longo das últimas décadas. As regiões mais inovadoras tendem a estar no topo ou próximas aos seus sistemas urbanos nacionais, em que as áreas metropolitanas estão entre os primeiros lugares a receber novos conhecimentos do exterior, recombiná-los em novos inventos e exportá-los em escala internacional (Simmie, 2001). Sendo assim, o debate acerca da integração dos agentes e aspectos inovadores ganhara espaço nas discussões das redes locais de inovação (Dicken, 2004; Saxenian, 1994) e das redes globais (Curran e Blackburn, 1994).

Aspectos preponderantes para a produção e assimilação de conhecimento advêm de fatores como a realização de pesquisas privadas e universitárias, os gastos em atividades de P\&D, o investimento em capital humano, o papel desempenhado pelas instituições, bem como as redes e estratégias corporativas e a composição demográfica das regiões (Crescenzi e Rodríguez-Pose, 2012). Todos esses fatores colaboram conjuntamente para propiciar a atividade inventiva, sendo que muitos deles requerem tempo para que sejam constituídos em determinadas localidades e, por sua vez, exercerão influência de longo prazo na perpetuação da criatividade. Porém, assim como proposto por Ó hUallacháin et al. (2015), novos insights acerca do processo inventivo das cidades surgem no escopo da Nova Geografia Econômica conforme a literatura avança. É exatamente essa a razão das hipóteses testadas ao longo deste trabalho, que busca fazer um paralelo dessa investigação para o Brasil em contraste 
aos resultados obtidos para um país desenvolvido.

Nesse âmbito, a produção de conhecimento e as invenções tecnológicas estão associadas aos diferentes atributos que caracterizam as regiões, podendo diferentes localidades serem geradoras de tecnologias com perfis similares, originando, assim, a primeira hipótese deste trabalho.

H1: há uma associação positiva entre o número de patentes em uma determinada classe tecnológica ${ }^{1}$ e o número de regiões que geram as patentes nessas classes.

Desde autores clássicos como Lösch (1955), argumenta-se que, ao se desconsiderarem as particularidades inerentes a cada região, a concentração da atividade econômica pode ser justificada por duas forças: retornos crescentes de escala e custos de transporte. Retornos crescentes de escala levam à maior concentração, enquanto os altos custos de transporte elevam o número de produtores. Na literatura mais recente, Feldman e Florida (1994) afirmam que, para que uma região gere invenções, essa deve possuir uma escala mínima, assim como características locais, como disponibilidade de mão de obra, fornecedores, disponibilidade de recursos e transporte influenciam a concentração da atividade tecnológica.

H2: o número de regiões que geram patentes em determinada classe tecnológica é negativamente associado ao tamanho da região.

Ainda com relação à concentração da atividade produtiva, essa pode se dar em determinada região devido às possibilidades de economias externas, incluindo, assim, fatores de produção comuns como terra, trabalho, capital, energia, sistemas de esgoto e transporte (Marshall, 1985). Esse conjunto de fatores possibilita maior grau de especialização que, por sua vez, aumenta a tendência para diminuir os preços dos fatores ou aumentar a produtividade. As grandes áreas metropolitanas estão repletas de inventores interagentes, empresas focadas na inovação, estruturas sociais que favorecem o pensamento não convencional e sistemas industriais que incentivam a formação de pequenas empresas dinâmicas (Ó hUallacháin et al., 2015). Observa-se que regiões metropolitanas possuem mais inventores do que locais de menor escala urbana e que as mesmas regiões geram mais patentes, indicando um retorno crescente de escala em função do tamanho da cidade (Boschma, 2005). A escala urbana favorece a formação de redes (networking) entre os indivíduos (Breschi e Lissoni, 2009; Miguelez e Moreno, 2013), assim como de conexões entre indivíduos e firmas, trocas de experiências a partir da mobilidade (Almeida e Kogut, 1999) spin-offs de trabalhadores (Muendler e Rauch, 2012), criação de startups (Brenner, 2004; Hall e Lerner, 2010) e papel do empreendedorismo na atividade inventiva (Audretsch e Keilbach, 2005). Daqui derivam nossas próximas hipóteses, que relacionam a diversidade inventiva, o porte da região e a produção tecnológica.

H3: o número de patentes em determinada classe tecnológica é negativamente

\footnotetext{
${ }^{1}$ As classes e subclasses das patentes se referem ao segundo e terceiro níveis hierárquicos de classificação internacional das patentes, segundo a Organização Mundial de Propriedade Intelectual.
} 
relacionado ao tamanho da região.

H4: a frequência de regiões geradoras de uma determinada classe tecnológica é inversamente relacionada ao tamanho médio das regiões que geram essa classe tecnológica.

H5: o número de patentes em determinada classe tecnológica é inversamente relacionado com o tamanho médio das regiões geradoras dessas patentes.

A relação entre patentes e escala urbana, evidenciada no trabalho de Ó hUallacháin et al. (2015) para os Estados Unidos, demonstra os efeitos da externalidade da urbanização concentrando a atividade inventiva nos maiores centros urbanos. Por meio dessa relação, pode-se observar a importância da urbanização para o sucesso ou declínio do processo inventivo. As regiões que comportam maior diversidade econômica tendem a ter maiores níveis de inovação. Isso se deve ao fato de que a presença de diferentes indústrias permite a reconfiguração do conhecimento adquirido, aumentando a propensão dos indivíduos em desenvolver novos produtos, diferenciados (Jacobs, 1969).

Jacobs (1969) descreve a diversidade urbana como estímulo à criatividade e à atividade econômica. A diversidade promove a interação humana e a troca de ideias entre setores industriais, ao demonstrar que as cidades de maior tamanho geram invenções mais sofisticadas tecnologicamente e não comuns aos pequenos centros, como retrata Simmie (2001) na afirmação de que o tamanho da região e a diversidade de invenções caminham juntos.

A teoria seminal dos lugares centrais de Christaller (1966) aponta como as atividades econômicas se distribuem entre as localidades centrais e como essas localizações possuem diferentes niveis de centralidade, sujeitos a uma hierarquia urbana que determina quais bens serão ofertados pelos diferentes centros urbanos. Em uma hierarquia urbana, as localidades mais desenvolvidas oferecem oportunidades diferenciadas aos inventores, podendo explorar soluções menos comuns por estarem situadas a níveis superiores de centralidade, usufruem de economias de aglomeração particulares, compartilhando facilidades de transporte, infraestrutura, entre outros serviços. Em trabalhos mais recentes, está sendo estabelecida a noção de que há um grupo de agentes criativos que se concentram em centros urbanos, de modo que, quanto maior o nível de centralidade, maiores são as capacidades inventivas daquela região (Lorenzen e Andersen, 2009).

Dessa forma, as regiões metropolitanas favorecem o processo inventivo, pois concentram em si características sociais locais que propulsionam a criatividade dos agentes. A constituição de redes inovadoras em grandes regiões urbanas atrai mais profissionais a se tornarem inventores e migrarem para essas regiões ao encontro de maiores oportunidades de interação entre profisionais qualificados (Bettencourt, 2013). Exatamente por isso espera-se dessas regiões a produção e a distribuição de bens que não se notam nas menores localidades, ressaltando sua maior diversidade funcional, 
conforme as hipóteses a seguir:

H6a: áreas com maior população são mais diversificadas tecnologicamente.

H6b: o total de patentes de uma região é positivamente relacionado com o número de classes de patentes na região.

H7a: quanto maior a região, maior o número médio de patentes por classes tecnológicas.

H7b: quanto maior o total de patentes de uma região, maior o número médio de patentes por classe tecnológica.

Essas últimas hipóteses, que relacionam o tamanho da região à sua capacidade inventiva, baseiam-se no o argumento de Malecki e Varaiya (1987). Segundo os autores, grandes áreas urbanas se caracterizam por taxas mais altas de inovação, adoção mais rápida de inovações e proporções mais altas de trabalhadores qualificados do que locais menores. O processo de invenção é, em termos gerais, um processo coletivo que envolve indivíduos em um grupo, firmas e organizações, fontes locacionais e meios de interação (Boschma, 2005). A aglomeração de trabalhadores qualificados em um mercado de trabalho ou um polo industrial em uma localidade são exemplos de fenômenos capazes de potencializar o processo criativo a partir do contato interpessoal (Acs, 2003; Carlino et al., 2007).

Diante de tais hipóteses apresentadas por Ó hUallacháin et al. (2015), que refletem as novas perspectivas sobre o processo inventivo das cidades, também abordados pela Nova Geografia Econômica, discutidas pelo autor em diálogo com a literatura, na próxima seção, são apresentados os dados e a metodologia empírica do trabalho, que será aplicada à análise da distribuição regional da atividade inventiva brasileira.

\section{Dados e Metodologia}

Os dados de patentes utilizados neste trabalho foram obtidos da Base de Dados Estatísticos de Propriedade Intelectual, versão 1.0, gerada pelo Instituto Nacional de Propriedade Industrial (BADEPI/INPI), referente ao período de 2000 a 2011 . O conjunto de dados coletados abrange 633 classes de patentes. Por outro lado, a unidade regional de análise é obtida a partir do conceito de região de influência dos centros urbanos (REGIC) (IBGE, 2008) que permite analisar as redes urbanas associadas aos principais centros urbanos de inovação, considerados redes de influência.

A utilização de classes de patentes como proxy para mensurar a atividade tecnológica pode ser considerada apropriada uma vez que é possivel rastrear a localidade dos inventores por meio dos dados de patentes. Patentes são usadas muito frequentemente como uma medida aproximada para a atividade inventiva. Como abordado por Pakes e Griliches (1984), as patentes são um bom indicador para a atividade inventiva, pois há forte relação entre $P \& D$ e o número de patentes. Porém, apesar da 
sua ampla utilização, as patentes não são medidas perfeitas para inovação, uma vez que se desconsidera o fato de que nem toda invenção ou inovação são patenteáveis (Nagaoka, 2010).

Para especificar a escala de uma região, serão utilizados dados de população das 392 regiões. As informações populacionais serão extraídas do Censo do Instituto Brasileiro de Geografia e Estatística (IBGE) de 2010. Essas estão segregadas a partir das Regiões de Influência das Cidades (REGIC). O corte regional a partir das REGIC, representado na Figura 1, foi utilizado nesta pesquisa por se tratar de um conceito construído de modo a refletir os fluxos no interior de regiões polarizadas e polarizadoras (Rodriguez e Gonçalves, 2017). ${ }^{2}$

Figura 1. Distribuição espacial das frequências de subclasses tecnológicas dos depósitos de patentes brasileiros, segundo o conceito de Regiões Intermediárias da Divisão Urbano-Regional da REGIC (2000-2011)



Fonte: Rodriguez e Gonçalves (2017).

\footnotetext{
${ }^{2}$ Rodriguez e Gonçalves (2017, p. 238-239) exemplificam a relevância desse corte regional: "A região intermediária de Campinas, por exemplo, possui 10.272 frequências de classificação de patentes, sendo que desse total somente 4.447 (43\%) são efetivamente da cidade de Campinas. Parte dessas frequências é atribuída a um grupo de municípios como Piracicaba, Vinhedo, Limeira, Valinhos e Americana (27\%). Em contraste, a região intermediária do Rio de Janeiro, que possui 9.177 frequências de códigos IPCs, registra somente na cidade do Rio de Janeiro 6.955 ocorrências, representando $75 \%$ da região, sendo outra parcela significativa assumida pelos municípios de Niterói e Petrópolis".
} 
O modelo empírico deste trabalho toma como referência Ó hUallacháin et al. (2015). De acordo com o referencial adotado, são testadas algumas hipóteses sobre a relação entre escala urbana e capacidade inventiva, utilizando-se de dados de classes de patentes como uma aproximação para a capacidade inventiva e dos tamanhos populacionais como aproximação para a escala urbana. A partir dessas hipóteses, avaliam-se relações lineares e não lineares entre as variáveis. As relações não lineares são importantes para verificar a intensidade da relação entre as variáveis, ou seja, se $Y$ cresce ou decresce a taxas crescentes ou decrescentes. Ao final desta seção, no Quadro 1, encontra-se um resumo das equações que são descritas a seguir, com as relações esperadas para cada regressão estimada.

A primeira equação pretende demonstrar a relação entre a atividade inventiva, estimada pelas classes de patentes, e a região geradora dessa invenção Ó hUallacháin et al. (2015). A variável dependente, REGIC, foi obtida por meio da contagem de regiões que geravam uma patente específica. Com base nisso, obteve-se uma medida de abrangência daquele tipo de inovação. Já a variável PATCL foi construída a partir da soma dos registros de patentes do INPI para cada classe de patente específica, produzidas pelas 392 regiões brasileiras. Todas as variáveis estão convertidas em logaritmos naturais.

A segunda equação é uma derivação da primeira apresentada e relaciona o número de regiões que produz uma classe de patente específica com o tamanho da menor área na qual se observa essa patente em particular. O porte da região é determinado pelos dados do censo populacional do IBGE. A variável endógena, MENREG, é a menor região que gera patente na classe $i$, enquanto REGIC se refere à quantidade de regiões que produzem essa mesma classe de patente. O coeficiente negativo da regressão significa que, conforme o número de regiões geradoras de inovação aumenta, expande-se a participação de pequenas localidades no processo inventivo. Christaller (1966) apud Ó hUallacháin et al. (2015) já apresentava essa associação ao definir que regiões de diferentes tamanhos participam da atividade inventiva, mas apenas regiões de maior porte produzem tecnologias diferenciadas, que não são habituais às pequenas áreas, conforme retrata a Equação 3.

Mori et al. (2008) substituíram a variável de tamanho da menor área inventiva (MENREG), medida pela menor dimensão populacional, pela variável MEDREG, que estima o porte médio da área metropolitana em que a classe de patente $i$ é observada, conforme estima a Equação 4. A variável MEDREG é construída a partir do cálculo da média populacional das localidades que apresentavam patentes para determinada classificação. Similarmente à hipótese anterior, na Equação 5, o tamanho médio da região está relacionado ao número de patentes geradas em uma determinada classe. 


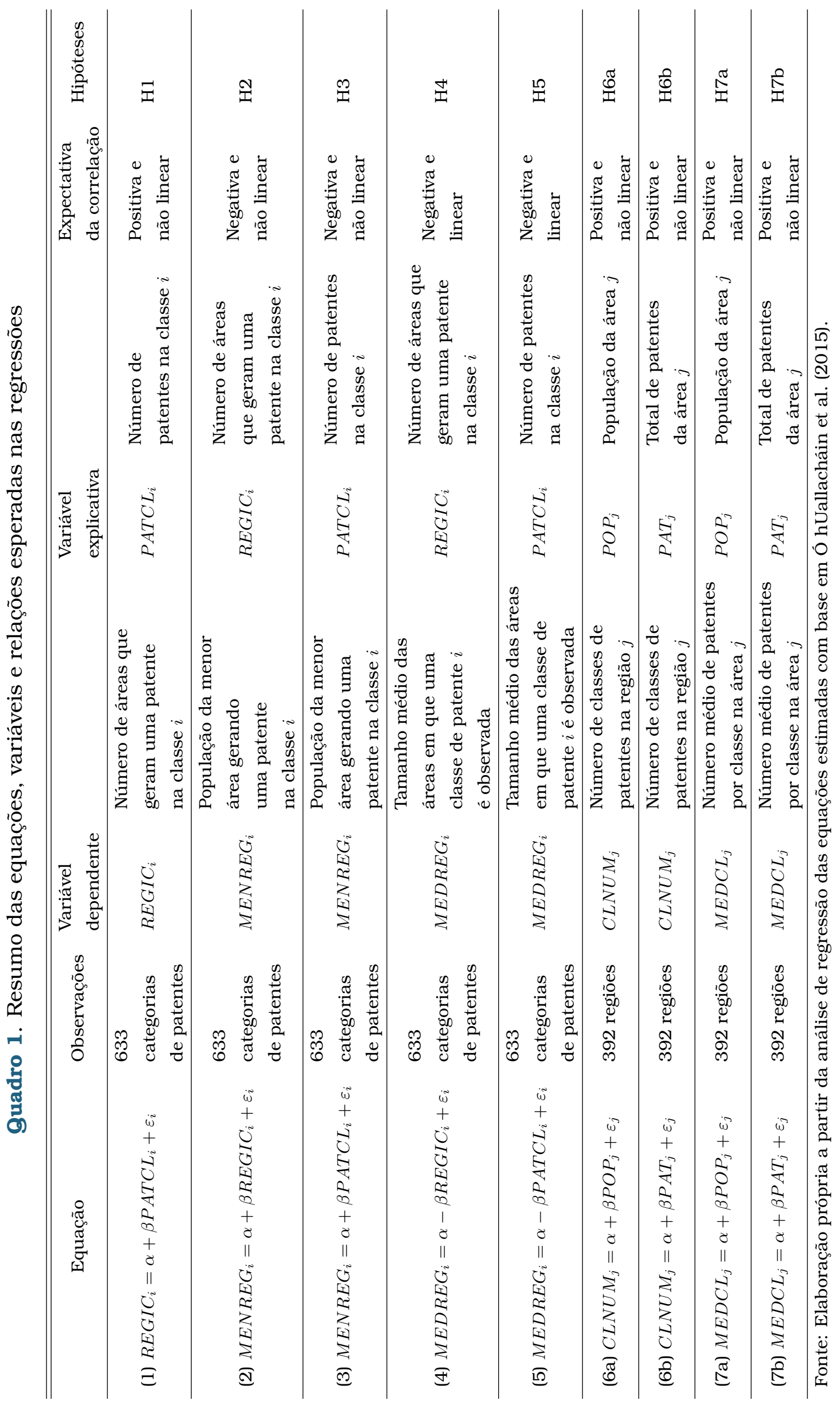


Também são analisadas possíveis relações entre o tamanho das regiões e o total de classes de patentes produzidas. A variável CLNUM, presente na Equação 6a, representa o número de classes de patentes presentes em uma região $j$ e foi obtida através da observação da quantidade de patentes de classes distintas produzidas dentro de uma região. A variável independente, POP, refere-se à população da área $j$. Pressupondo a hierarquia urbana de inovação, espera-se que o coeficiente $\beta$ seja menor do que 1, indicando que, diante de um aumento da população de determinada região, torna-se mais difícil acrescentar uma nova variedade de patente à quantidade de patentes produzidas por uma região de grande porte em comparação a uma de menor tamanho. Em um segundo momento, na Equação 6b, a variável POP foi substituída na expressão por PAT, referindo-se ao total de patentes. Dessa forma, pode-se mensurar o valor total das invenções (PAT), sendo por isso considerado um indicador mais abrangente da variedade tecnológica de uma região.

Outra equação é elaborada a partir da quantidade média de patentes por classe tecnológica, MEDCL, referente à contagem total de patentes de uma área específica dividida pela quantidade de classes. Espera-se que o valor de MEDCL seja maior para os maiores centros urbanos, refletindo a noção de que as regiões mais populosas também são as mais diversificadas, como representado pela Equação 7a. Analogamente ao que foi feito na Equação 6a, que originou a relação expressa em 6b, a Equação 7a também será alterada e dará origem à relação expressa na Equação $7 \mathrm{~b}$, em que a variável POP foi substituída por PAT. Nessa última relação, espera-se que regiões de grande escala de produção tecnológica sejam diversificadas, evidenciando que a variedade de códigos de patentes tende a ser maior em centros que geram maior número de patentes, conforme descrito pela hipótese H7b na seção 2.

\section{Resultados}

\subsection{Resultados descritivos}

A Tabela 1 fornece uma configuração inicial da distribuição de patentes por regiões a partir da média de patentes geradas por cada localidade e a quantidade de códigos de patentes apresentados, levando em consideração as dez regiões de maior influência no processo inventivo brasileiro. O tamanho médio das áreas metropolitanas que geram maior diversidade de patentes varia de 1,08 milhões de pessoas em Campinas a 11,2 milhões em São Paulo, sendo esta a região de maior porte.

Observa-se também que as maiores áreas dispõem de maior potencial inventivo, apresentando não apenas um volume de patenteamento superior às demais regiões como também maior variedade, abrangendo mais tipos de invenções, como demonstram os dados referentes às classes de patentes observadas para cada região. Em geral, o tamanho médio da população das dez regiões com maior dinamismo tecnológico excede o tamanho médio da população das dez regiões de menor diversificação inventiva, caracterizadas por patentes comuns amplamente distribuídas. Esse padrão 
se assemelha ao princípio da hierarquia de Christaller, de que os grandes centros são nós na provisão de funções incomuns de alta ordem.

Tabela 1. Regiões brasileiras com mais invenções patenteadas entre 2000 - 2011

\begin{tabular}{lrrrrr}
\hline \multicolumn{1}{c}{ Regiões } & UF & População & $\begin{array}{c}\text { Média de Patentes } \\
\text { por Classe }\end{array}$ & $\begin{array}{c}\text { Classes de } \\
\text { Patentes }\end{array}$ & $\begin{array}{c}\text { Total de } \\
\text { Patentes }\end{array}$ \\
\hline São Paulo & SP & 11.253 .503 & 36,86 & 586 & 21.602 \\
Rio de Janeiro & RJ & 6.320 .446 & 12,45 & 489 & 6.088 \\
Belo Horizonte & MG & 2.375 .151 & 10,19 & 476 & 4.851 \\
Curitiba & PR & 1.751 .907 & 11,12 & 461 & 5.127 \\
Campinas & SP & 1.080 .113 & 11,52 & 455 & 5.242 \\
Porto Alegre & RS & 1.409 .351 & 8,37 & 431 & 3.609 \\
São José dos Campos & SP & 629.921 & 4,46 & 346 & 1.545 \\
Florianópolis & SC & 421.240 & 4,77 & 341 & 1.627 \\
Brasília & DF & 2.570 .160 & 5,19 & 317 & 1.646 \\
Joinville & SC & 515.288 & 5,49 & 310 & 1.703 \\
Total & & 28.327 .080 & 110,42 & $4212^{*}$ & 53.040 \\
Brasil & BR & 143.256 .424 & 145,16 & 633 & 93.469 \\
\hline \hline
\end{tabular}

Fonte: Base de Dados Estatísticos de Propriedade Intelectual - Badepi/Inpi, v. 1.0; Instituto Brasileiro de Geografia e Estatística - IBGE. Elaboração própria.

*Não significa que sejam 4212 classes de patentes distintas, mas sim o total de categorias que cada uma das regiões somadas gera. ${ }^{* *}$ A lista de regiões da Tabela 1 não é exaustiva. Algumas que também possuem grande quantidade de patentes são Recife, São Carlos e Campina Grande, por exemplo.

\subsection{Resultados empíricos}

Seguindo o modelo de estimação adotado por Ó hUallacháin et al. (2015) ${ }^{3}$ e respeitando as particularidades dos dados de patentes coletados para as regiões brasileiras, pode-se inferir que algumas regressões possuem relações não lineares entre as variáveis em questão, enquanto em outras equações foram estabelecidas relações lineares entre as variáveis observadas. Nesse sentido, serão testadas as equações que suscitam possiveis relações entre invenção e hierarquia urbana das Regics. Conforme apresentado no Guadro 1, serão analisadas as relações expressas pelas funções de 1 a 7b, buscando verificar o comportamento esperado das variáveis nas regressões estimadas. Os resultados obtidos para essas regressões são apresentados Tabela 2.

\footnotetext{
${ }^{3} \mathrm{O}$ autor referido utiliza dados de patentes concedidas, enquanto neste artigo se trabalha com dados de depósitos de patentes.
} 
Tabela 2. Equações de regressão de 1 a 7b

\begin{tabular}{|c|c|c|c|c|}
\hline Equações & $R^{2}$ & $\mathrm{~F}$ & $p-v a l o r$ & $\mathrm{~N}$ \\
\hline $\begin{array}{c}\text { 1) } R E G I C_{i}=-0,07 P A T C L_{i}+0,88 P A T C L_{i}+0,03 \\
(0,01)^{* * *}(0,019)^{* * *}(0,02)^{*}\end{array}$ & 0,97 & 9363,01 & 0,00 & 644 \\
\hline 2) $\begin{aligned} M E N R E G_{i}= & 0,17 R E G I C_{i}-1,72 R E G I C_{i}+14,39 \\
& (0,02)^{* * *}(0,09)^{* * *}(0,11)^{* * *}\end{aligned}$ & 0,70 & 736,73 & 0,00 & 644 \\
\hline 3) $\begin{aligned} M E N R E G_{i}= & 0,10 P A T C L_{i}-1,29 P A T C L_{i}+14,26 \\
& (0,01)^{* * *}(0,07)^{* * *}(0,12)^{* * *}\end{aligned}$ & 0,64 & 559,12 & 0,00 & 644 \\
\hline 4) $\begin{aligned} M E D R E G_{i}=- & 0,466 R E G I C_{i}+6,76 \\
& (0,02)^{* * *}(0,02)^{* * *}\end{aligned}$ & 0,55 & 768,49 & 0,00 & 633 \\
\hline 5) $\begin{aligned} M E D R E G_{i}= & -0,303 P A T C L_{i}+6,67 \\
& (0,01)^{* * *}(0,02)^{* * *}\end{aligned}$ & 0,51 & 666,35 & 0,00 & 633 \\
\hline 6a) $\begin{aligned} C L N U M_{j}= & 1,02 P O P_{j}+2,88 \\
& (0,06)^{* * *}(0,30)^{* * *}\end{aligned}$ & 0,44 & 300,39 & 0,00 & 392 \\
\hline 6b) $\begin{aligned} C L N U M_{j}=- & 0,14 P A T_{j}^{2}+1,33 P A T_{j}+0,66 \\
& (0,01)^{* * *}(0,02)^{* * *}(0,01)^{* * *}\end{aligned}$ & 0,99 & 13343,19 & 0,00 & 392 \\
\hline $\begin{aligned} \text { 7a) } M E D C L_{j}= & 0,21 P O P_{j}{ }^{2}-1,945 P O P_{j}+4,52 \\
& (0,01)^{* * *}(0,15)^{* * *}(0,38)^{* * *}\end{aligned}$ & 0,67 & 392,59 & 0,00 & 392 \\
\hline 7b) $\begin{aligned} M E D C L_{j}= & 0,12 P A T_{j}^{2}-0,26 P A T_{j}+0,114 \\
& (0,00)^{* * *}(0,01)^{* * *}(0,01)^{* * *}\end{aligned}$ & 0,95 & 3966,43 & 0,00 & 392 \\
\hline
\end{tabular}

Fonte: Elaboração própria.

Nota: ***Significância a 1\%; ** Significância de 5\%; * Significância de $10 \%$.

Na primeira equação testada, observa-se a relação de não linearidade entre a quantidade de patentes de determinada classe e a quantidade de regiões que geram essa invenção, o que evidencia que tal correlação não se dá de forma proporcional. Ou seja, espera-se que $\beta$ (coeficiente da regressão) seja menor do que 1 , demonstrando que a quantidade de localidades que geram classes de patentes aumente a taxas decrescentes (Ó hUallacháin et al., 2015). Esse resultado indica que há um padrão similar ao identificado por Ó hUallacháin et al. (2015) para os Estados Unidos no caso brasileiro, contudo é possivel notar que, no caso brasileiro, a concentração de classes de patentes é maior nos maiores centros urbanos, o que reflete em um coeficiente linear maior para o caso brasileiro (0,8815 comparado ao 0,398 do resultado para os EUA). Dessa forma, a Figura 1 representa graficamente a regressão retratada na Equação 1. 
Figura 1. Relação entre o número de regiões gerando uma patente na classe $i$ (REGIC) e o número total de patentes na classe $i$ (PATCL)

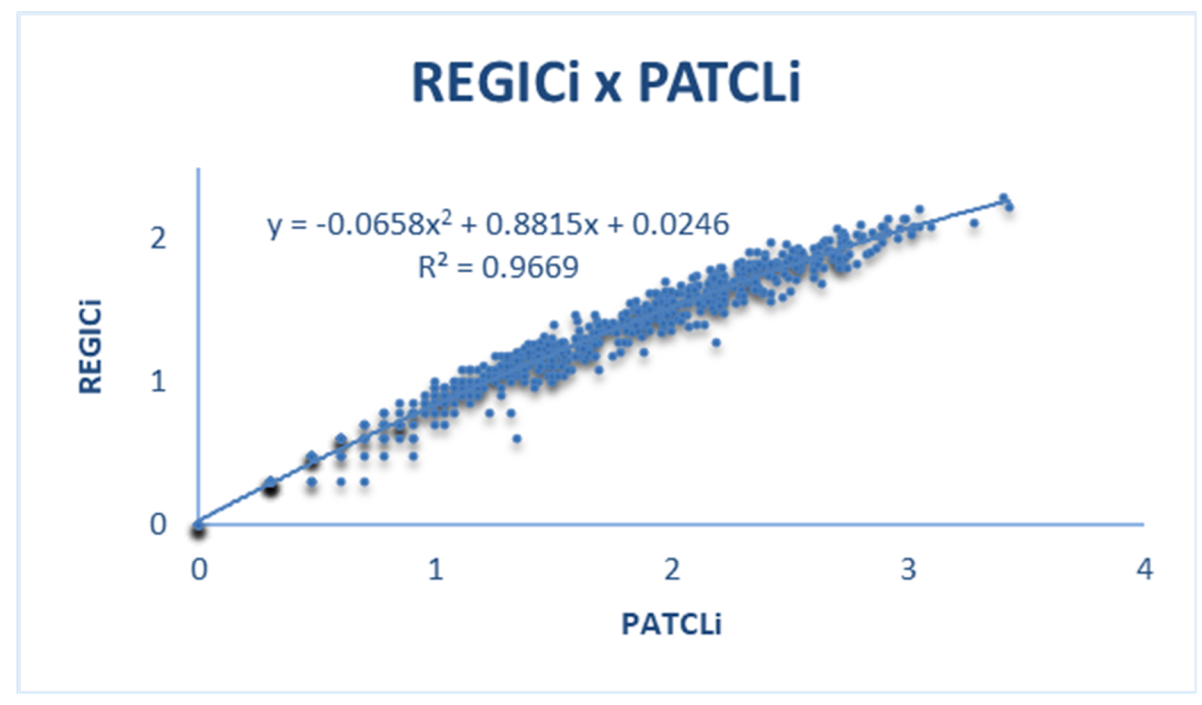

Fonte: Elaboração própria.

Para a Equação 1, os resultados indicam uma correlação positiva entre a quantidade de determinada patente e a frequência de regiões que geram essa invenção, conforme o esperado pela hipótese $\mathrm{H} 1$ descrita na seção 2. Isso significa que, quando aumenta o número de patentes em uma determinada classe $i$, o número de regiões que geram tal tecnologia também aumenta, embora a uma taxa decrescente, conforme demonstra a não linearidade entre os parâmetros. Os valores se mostram significativos a uma significância de 5\%.

Nas duas regressões que seguem, nota-se novamente a presença de não linearidade entre elas. A primeira regressão representa a Equação 2 contida no Quadro 1, relacionando o tamanho da Regic geradora de determinada invenção e a quantidade de Regics que originam essa mesma patente. Já a regressão representada pela Equação 3 correlaciona a quantidade de patentes pertencentes a uma mesma classe tecnológica e o tamanho das Regics que originam essas patentes, testando a Hipótese 3 , de que invenções mais comuns se restringem a pequenas localidades.

A Equação 2 apresenta uma relação negativa entre a região de menor porte geradora de invenção em uma determinada classe de patentes $i$ e o número de regiões que geram tal patente na classe $i$, com um coeficiente de -1,718, enquanto nos EUA o coeficiente possui valor de -0,257 (Ó hUallacháin et al., 2015). Isso significa que o aumento do número de regiões que geram determinada tecnologia ocorre conjuntamente à diminuição do tamanho populacional da região, sugerindo ubiquidade tecnológica. No Brasil, porém, o número de áreas que geram determinada tecnologia, para uma dada população, cai mais rapidamente do que nos EUA, sugerindo que áreas urbanas pequenas patenteiam relativamente menos. 
Figura 2 e 3. Associação da população da menor área urbana gerando uma patente na classe $i\left(M E N R E G_{i}\right)$ com o número de áreas urbanas que geram patentes na classe $i\left(R E G I C_{i}\right.$, Equação 2) e com a quantidade de patentes na classe $i$ ( $\left.P A T C L_{i}\right)$

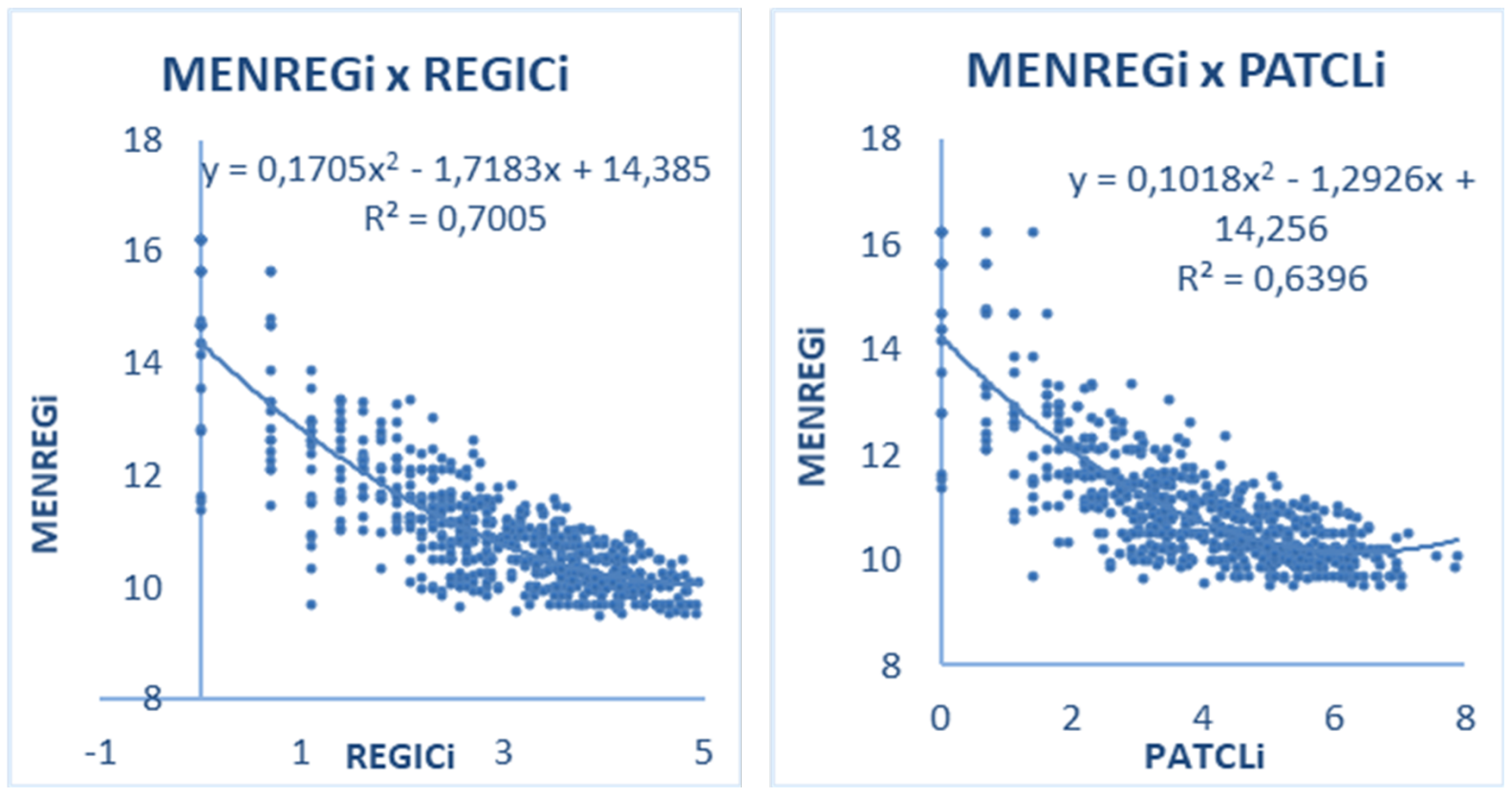

Fonte: Elaboração própria.

Analogamente ao verificado na regressão anterior, a terceira equação apresenta coeficiente negativo e estatisticamente significativo $(-1,29)$ para a variável explicativa $\left(P A T C L_{i}\right)$, o que significa que, quanto maior o número de patentes em determinada classe tecnológica, menor é o tamanho, em média, das regiões que geram tais patentes. Isso justifica a hipótese levantada neste trabalho de que patentes mais usuais são geradas tanto por regiões de maior porte, mas principalmente estas são muito comuns a regiões de menor dimensão urbana. O fenômeno é bem mais forte do que nos EUA, em que o coeficiente possui valor de -0,156 (Ó hUallacháin et al., 2015). A Equação 4 reafirma essa hipótese ao mostrar uma relação negativa entre o número de regiões que geram uma patente na classe $i$ e o tamanho médio das regiões geradoras desse tipo de invenção.

A Figura 4 representa a relação entre a quantidade de regiões que geram uma patente na classe $i$ e o porte médio dessas regiões, corroborando a hipótese já vista nesta seção e na seção 2 de que as regiões de maior porte produzem invenções menos comuns às demais localidades. Já a regressão representada na Figura 5 demonstra a relação entre o número de patentes observadas em uma classe $i$ e o tamanho médio das regiões em que se observa essa mesma classe de patente. As relações mostradas nas Figuras 4 e 5 são também observadas nos EUA (Ó hUallacháin et al., 2015).

A Regressão 5 estabelece uma relação negativa entre o número de patentes da classe $i$ e a escala urbana da região em que essa mesma categoria de patente é observada. Isso aponta que, quanto mais frequente uma classe de patente se torna, mais 
provável será de se encontrar tal classe tecnológica em regiões de menor tamanho populacional. Há, no entanto, observações discrepantes, nos casos em que regiões menores geram patentes pouco frequentes, como acontece em Botucatu e Seabra, por exemplo. Diferentemente das demais regressões analisadas até aqui, as Equações 4 e 5 apresentam relações lineares.

Figura 4 e 5. Associação do tamanho médio da população das áreas em que a classe de patente $i$ é observada $\left(M E D R E G_{i}\right)$ com o número de áreas gerando patentes na classe $i\left(R E G I C_{i}\right)$ - à esquerda. Relação entre o tamanho médio da população das áreas metropolitanas em que a classe de patente $i$ é observada ( $M E D R E G_{i}$ ) com número de patentes metropolitanas na classe $i\left(P A T C L_{i}\right)$ - à direita
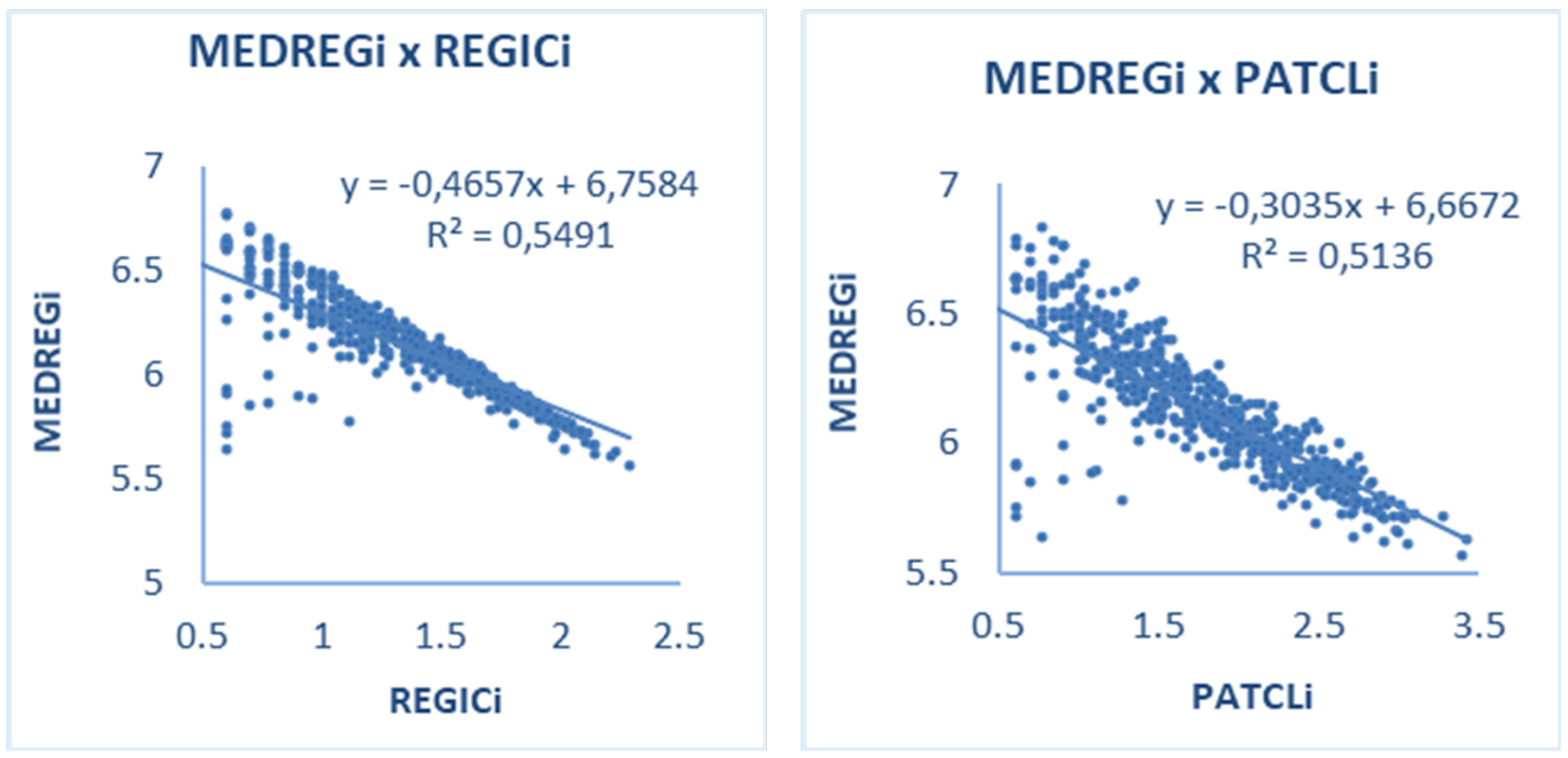

Fonte: Elaboração própria.

As Equações 6a e 6b são representadas graficamente pelas Figuras 6 e 7 a seguir. Observa-se linearidade na Equação 6a e não linearidade na Equação 6b. A Regressão 6a estabelece a relação positiva entre a população da região e a variedade de classes de patentes na região, servindo de mensuração da diversidade inventiva das mesmas regiões. No caso brasileiro, a relação é crescente, mas nos EUA há uma taxa de crescimento que passa a decrescer quando a população é muito grande (Ó hUallacháin et al., 2015). Tal diferença de resultados sugere que as invenções são muito concentradas nos grandes centros brasileiros, os quais dominam várias classes de patentes.

Já a regressão representada em 6 b revela uma relação positiva entre o total de patentes da região e o número de classes de patentes da região, também sinalizando que regiões mais patenteadoras são também em média regiões com competências tecnológicas mais diversificadas, embora tal relação tenda a crescer a taxas decrescentes. Esse mesmo tipo de padrão ocorre nos EUA (Ó hUallacháin et al., 2015), com as diferenças de magnitudes dos coeficientes, sendo maiores no Brasil. 
Figura 6 e 7. Figura 6 (à esquerda) mostra a associação do número de classes de patentes na área $j\left(C L N U M_{j}\right)$ e a população da área $j\left(P O P_{j}\right)$ (Equação 6a). Figura 7 (à direita) mostra a associação do total de classes de patentes geradas na área $j$ $\left(C L N U M_{j}\right)$ e o número de patentes ( $\left.P A T_{j}\right)$ (Equação 6b)
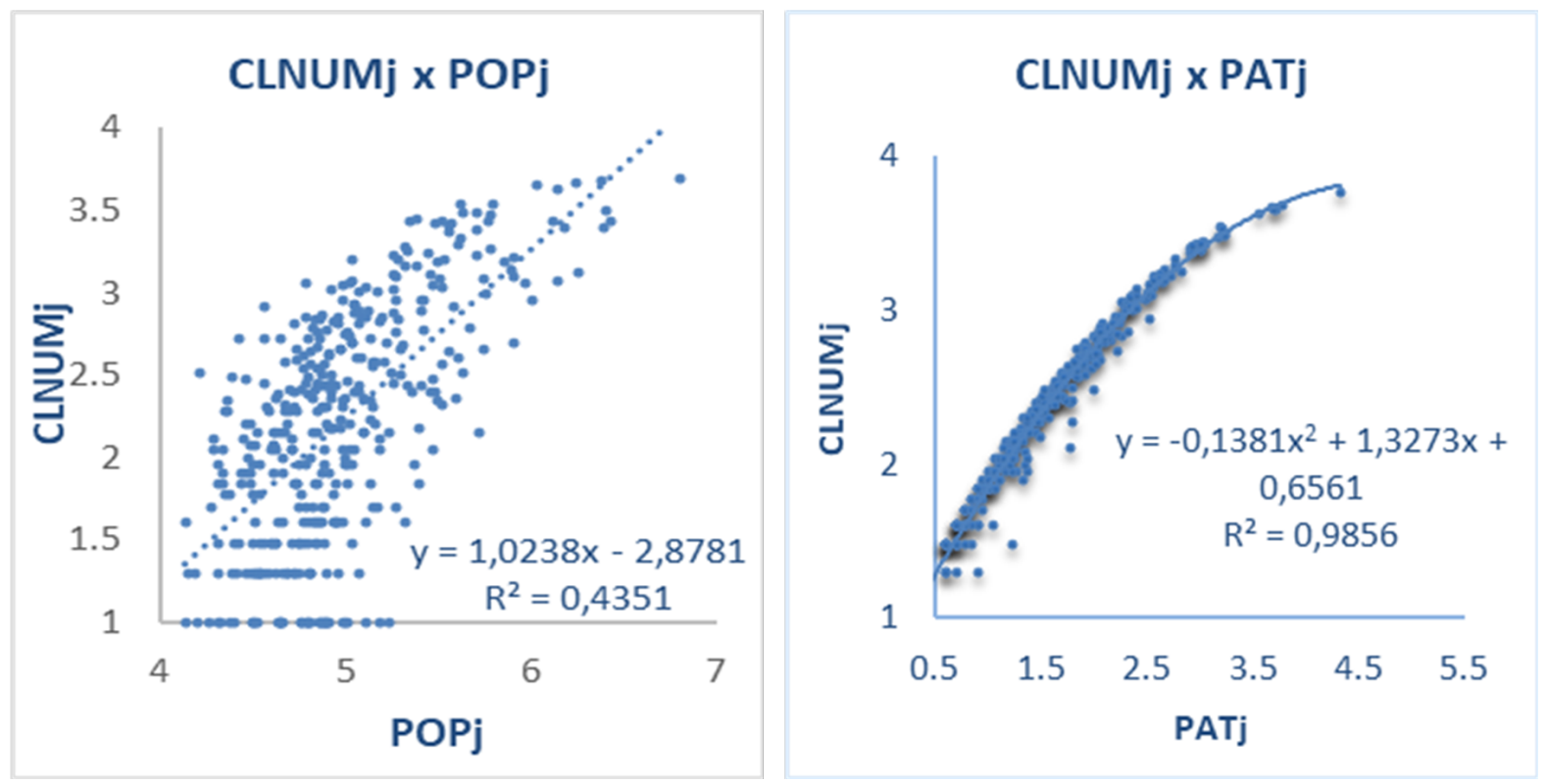

Fonte: Elaboração própria.

Nota-se que há uma correlação entre o porte da região, mensurado pela densidade populacional, e a quantidade de classes de patentes distintas. A segunda equação demonstrou uma relação positiva entre o total de patentes da área j e o número de classes de patentes na região $j$, sinalizando que regiões que mais produzem invenção são também, em média, regiões com competências tecnológicas mais diversificadas.

Os fatos estilizados da teoria de Christaller (1966) também associam, indiretamente, tamanho metropolitano e diversidade econômica. É praticamente improvável que pequenas regiões com capital humano menos diversificado contribuam para formas incomuns de avanço tecnológico, ou seja, invenções raras habitualmente se restringem aos locais de maior escala urbana. O número de classes de patentes observadas em uma região provavelmente aumentará com o tamanho metropolitano. $\mathrm{O}$ porte também deve influenciar o número médio de patentes por classe em todas as regiões, com regiões menores gerando menos patentes na classe média.

É nítida, portanto, a evidência da relação entre as regiões de maior porte no sistema de inovação brasileiro e a diversidade das classes de patentes geradas pelas mesmas regiões. É de se esperar que as regiões de maior dinamismo tecnológico não apenas possuam maior diversidade criativa e inventiva, como também originem mais depósitos de patentes.

As Equações 7a e 7b avaliam a relação entre o tamanho populacional (POP) e o número médio de patentes por classe na região $j\left(M E D C L_{j}\right)$ e a relação entre o total 
de patentes da região j (PAT) e o número médio de patentes por classe na região $j$, respectivamente. Ambas estabelecem relações de não linearidade entre os termos. Nas Figuras 8 e 9 abaixo, observa-se o número médio de patentes por classe associado à população da região inventora e ao total de patentes da mesma, respectivamente. Em ambos os gráficos, a relação é positiva entre as variáveis, confirmando as hipóteses descritas na seção 2 .

Figura 8 e 9. Figura 8 (à esquerda) mostra a associação do número médio de patentes por classe gerada na área j $\left(M E D C L_{j}\right)$ e sua população $\left(P O P_{j}\right.$; Equação 7a). Figura 9 (à direita) mostra a associação do número médio de patentes por classe gerada na área j $\left(M E D C L_{j}\right)$ e o número de patentes $\left(P A T_{j}\right)$ (Equação $\left.7 \mathrm{~b}\right)$
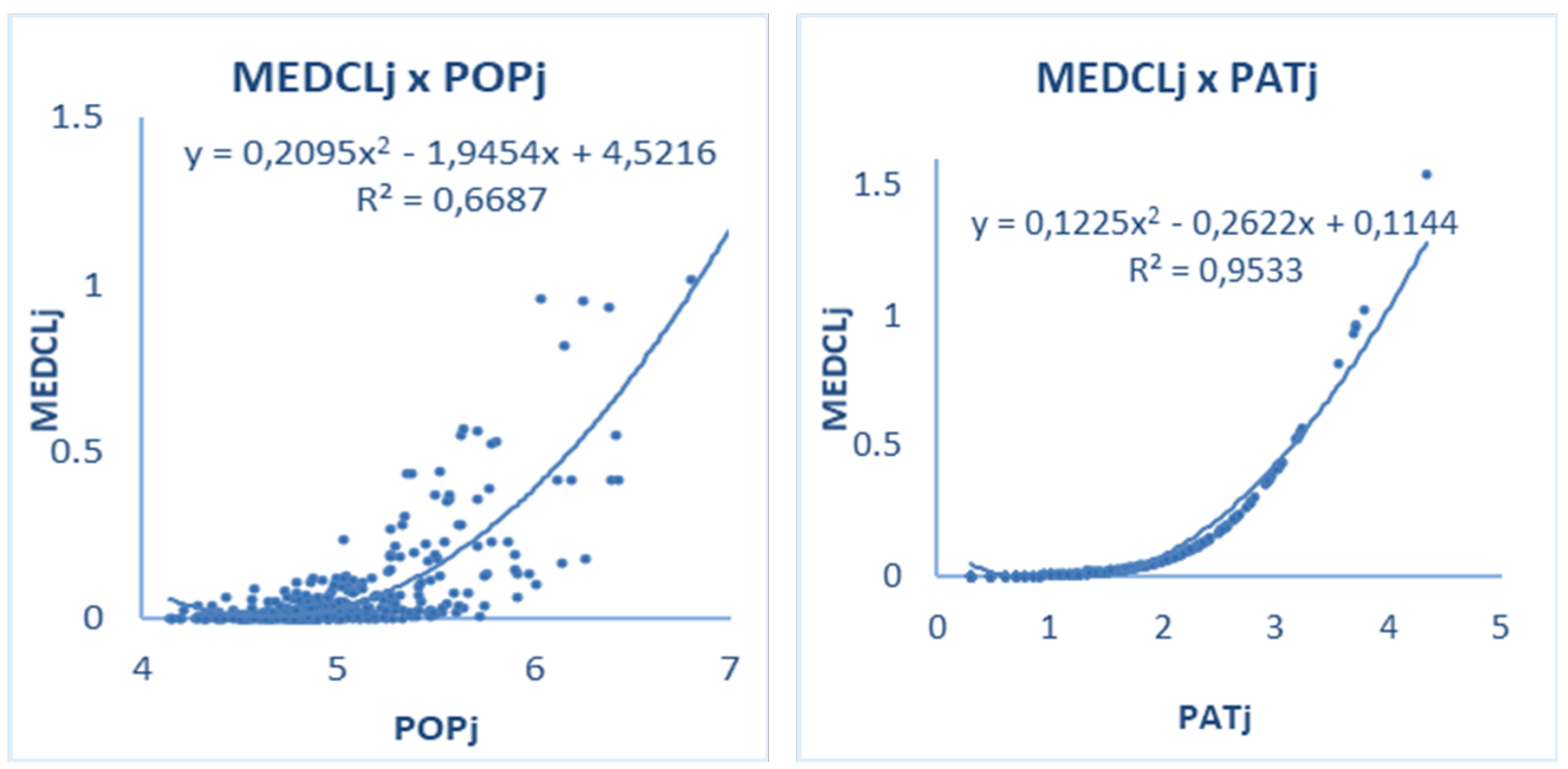

Fonte: Elaboração própria.

Ambos os coeficientes nas figuras acima são positivos e significativos, sugerindo que o número médio de patentes por região é maior quanto maior for a região e que, com o aumento da escala tecnológica da região, o número médio de patentes por classe também aumenta, embora o resultado seja consistente com os resultados anteriores que mostram um número desproporcional de patentes sendo gerado por inventores nas maiores cidades (Ó hUallacháin et al., 2015). À medida que as atividades de patenteamento aumentam, há uma expansão direta no número médio de patentes por classe, ou seja, a expansão é significativamente maior nas regiões maiores. No caso da Figura 7a, notam-se poucos casos de regiões com grande população que possuem alto número médio de patentes por classe. No caso americano (Ó hUallacháin et al., 2015), há uma frequência maior de áreas metropolitanas que demonstram maiores números médios de patentes por classes. Isso denota que a invenção é muito desigualmente distribuída no caso brasileiro, tanto em termos de magnitude (escala inventiva) quanto em termos de diversidade de classes. 


\section{Conclusões}

Este artigo investigou a atividade inventiva no Brasil, para o período de 2000 a 2011 , por meio da análise dos depósitos de patentes do INPI, discutindo a distribuição da invenção dentre as microrregiões brasileiras e a hierarquia urbana de inovação do país. Os resultados obtidos apontaram a concentração desproporcional de invenção nas regiões de maior porte e identificaram especializações tecnológicas em cidades e regiões específicas.

A distribuição desigual da invenção está ligada à organização hierárquica do sistema da cidade, como retratada na teoria dos lugares centrais de Christaller (1966). Há evidência de que as classes de patentes mais habituais se alastram pelas diversas regiões, indicando que o tamanho médio desses lugares é significativamente menor em comparação com as áreas que geram invenções incomuns, mais raras.

Essas áreas mais amplas são geralmente mais diversificadas em suas contribuições para o avanço tecnológico, especialmente quando a quantidade de patenteamento inventivo é empregada para medir o tamanho do centro urbano. O número médio de patentes por classe também é maior nas regiões de maior porte. Com os resultados obtidos nesta pesquisa, chegou-se à conclusão de que esses foram consistentes com os trabalhos de Ó hUallacháin et al. (2015); Simões et al. (2010) e que, portanto, as maiores regiões têm muitas funções, as pequenas localidades têm poucas e as grandes mesclam as funções de pequenas áreas e outras atividades adicionais.

Com relação à localização e à organização espacial da invenção, assim como demonstrado por Rodriguez e Gonçalves (2017), há forte concentração espacial da atividade inventiva, capaz de influenciar os resultados alcançados. Os padrões tecnológicos na região Sudeste são diferenciados, apresentando regiões de maior dinamismo inventivo, concentrando não apenas as atividades inventivas, mas também maior pluralidade de categorias de patentes.

Os atributos característicos às regiões que promovem invenção e especialização tecnológica sugerem que a posição na hierarquia urbana é eventualmente tão importante para as categorias de invenção quanto a singularidade espacial, institucional e social de uma região. Esses fatores podem ajudar a explicar por que algumas relações hierárquicas não são perfeitas. O fato de atrelar aos inventores das grandes regiões a produção de patentes tecnológicas raras é um princípio relevante para a compreensão da vantagem de escala que um lugar pode usufruir para seu progresso inventivo. As habilidades e os interesses incomuns de muitos inventores das grandes cidades podem gerar externalidades positivas que beneficiam os inventores que trabalham em campos comuns e raros (Ó hUallacháin et al., 2015). As maiores localidades propiciam melhores oportunidades de interação entre os agentes.

Comparando o caso brasileiro com o caso norte-americano, notam-se as mesmas

relações entre as variáveis número e tamanho das regiões e número de classes de 
patentes ou de patentes. A diferença é a intensidade das relações, com coeficientes de maiores valores absolutos no caso brasileiro. Em geral, isso denota que o fenômeno da invenção é mais espacialmente concentrado no Brasil, em termos da pequena frequência de regiões com maiores escalas inventivas e presença de diversidade de classes de patentes.

Sendo assim, a dimensão geográfica é um determinante importante das variedades de invenção que uma região pode gerar e de qual será o grau de envolvimento tecnológico dessa região dentro do sistema de inovação do país. Políticas urbanas que visem promover atividades criativas necessitam compreender a relevância desse fator no processo de inovação. Para isto, políticas precisam ser formuladas de acordo com as restrições que o tamanho impõe às capacidades e potencialidades de cada região. Investimentos que estimulem a produção de atividades de P\&D são necessários para o desenvolvimento de uma região e seu desempenho na hierarquia urbana de inovação.

\section{Referências}

Acs, Z. J. (2003). Innovation and the Growth of Cities. Edward Elgar Publishing.

Almeida, P. e Kogut, B. (1999). Localization of knowledge and the mobility of engineers in regional networks. Management Science, 45(7):905-917.

Araujo, V. D. C., Garcia, R., et al. (2016). Local determinants of innovation and spatial dependence: a spatial Tobit model applied to Brazilian micro-regions. In: Anais do XLII Encontro Nacional de Economia [Proceedings of the 42nd Brazilian Economics Meeting], number 161.

Audretsch, D. B. e Feldman, M. P. (1996). R\&D spillovers and the geography of innovation and production. The American Economic Review, 86(3):630-640.

Audretsch, D. B. e Keilbach, M. (2005). The mobility of economic agents as conduits of knowledge spillovers. In: The role of labour mobility and informal networks for knowledge transfer, Página 8-25. Springer.

Berger, P. L. e Luckmann, T. (1966). The social construction of reality: A treatise in the sociology of knowledge. Anchor.

Bettencourt, L. M. A. (2013). The origins of scaling in cities. Science, 340(6139):14381441.

Boschma, R. (2005). Proximity and innovation: a critical assessment. Regional Studies, 39(1):61-74.

Brenner, T. (2004). Local industrial clusters: existence, emergence and evolution. Routledge. 
Breschi, S. e Lissoni, F. (2009). Mobility of skilled workers and co-invention networks: an anatomy of localized knowledge flows. Journal of Economic Geography, 9(4):439468.

Carlino, G. A., Chatterjee, S., e Hunt, R. M. (2007). Urban density and the rate of invention. Journal of Urban Economics, 61(3):389-419.

Christaller, W. (1966). Central places in southern Germany. Prentice-Hall.

Crescenzi, R. e Rodríguez-Pose, A. (2012). An 'integrated'framework for the comparative analysis of the territorial innovation dynamics of developed and emerging countries. Journal of Economic Surveys, 26(3):517-533.

Curran, J. e Blackburn, R. (1994). Small firms and local economic networks: the death of the local economy? Paul Chapman.

Dicken, P. (2004). Global-local tensions: Firms and states in the global spaceeconomy. Reading Economic Geography, Página 137.

Feldman, M. P. e Florida, R. (1994). The geographic sources of innovation: technological infrastructure and product innovation in the United States. Annals of the Association of American Geographers, 84(2):210-229.

Feldman, M. P. e Kogler, D. F. (2010). Stylized facts in the geography of innovation. In: Handbook of the Economics of Innovation, volume 1, Página 381-410. Elsevier.

Fujita, M. e Mori, T. (2005). Frontiers of the new economic geography. Papers in Regional Science, 84(3):377-405.

Hall, B. H. e Lerner, J. (2010). The financing of R\&D and innovation. In: Handbook of the Economics of Innovation, volume 1, Página 609-639. Elsevier.

Howells, J. R. (2002). Tacit knowledge, innovation and economic geography. Urban Studies, 39(5-6):871-884.

IBGE (2008). Regiões de influência das cidades 2007. Instituto Brasileiro de Geografia e Estatística.

Jacobs, J. (1969). The economy of cities. New York: Vintage.

Krugman, P. (1996). Confronting the mystery of urban hierarchy. Journal of the Japanese and International economies, 10(4):399-418.

Lorenzen, M. e Andersen, K. V. (2009). Centrality and creativity: Does Richard Florida's creative class offer new insights into urban hierarchy? Economic Geography, 85(4):363-390.

Malecki, E. J. e Varaiya, P. (1987). Innovation and changes in regional structure. In: Handbook of Regional and Urban Economics, volume 1, Página 629-645. 
Marshall, A. (1985). Princípios de economia: tratado introdutório. Nova Cultural São Paulo.

Miguelez, E. e Moreno, R. (2013). Do Labour mobility and technological collaborations foster geographical knowledge diffusion? the case of European Regions. Growth and Change, 44(2):321-354.

Montenegro, R. L., Gonçalves, E., e Almeida, E. (2011). Dinâmica espacial e temporal da inovação no estado de São Paulo: uma análise das externalidades de diversificação e especialização. Estudos Econômicos, 41(4):743-776.

Mori, T., Nishikimi, K., e Smith, T. E. (2008). The number-average size rule: a new empirical relationship between industrial location and city size. Journal of Regional Science, 48(1): 165-211.

Muendler, M.-A. e Rauch, J. E. (2012). Mobilizing social capital through employee spinoffs. Texto para discussão, National Bureau of Economic Research.

Ó hUallacháin, B., Kane, K., e Kenyon, S. (2015). Invention in the United States city system. Annals of the Association of American Geographers, 105(6):1300-1323.

Rodriguez, R. S. e Gonçalves, E. (2017). Hierarquia e concentração na distribuição regional brasileira de invenções por tipos de tecnologias. Revista Brasileira de Inovação, 16(2):225-266.

Ruiz, R. M. (2005). Estruturas urbanas comparadas: Estados Unidos e Brasil. Estudos Econômicos (São Paulo), 35(4):715-737.

Saxenian, A. (1994). Regional networks: industrial adaptation in Silicon Valley and route 128. Cambridge, MA, Harvard University Press.

Scott, A. J. (2009). Human capital resources and requirements across the metropolitan hierarchy of the usa. Journal of Economic Geography, 9(2):207-226.

Simmie, J. (2001). Innovative cities. Taylor \& Francis.

Simões, R. F., do Amaral, P. V. M., Pereira, M. A., Cardoso, V. L., e Campos, S. M. C. (2010). Centralidades e hierarquia urbana em Minas Gerais: uma visão prospectiva. XIV Seminário sobre a Economia Mineira. Anais... Belo Horizonte: Cedeplar, Universidade Federal de Minas Gerais.

(๔) Este artigo está licenciado com uma CC BY 4.0 license. 